\title{
Cortico-Thalamo-Cortical Circuits of Mouse Forelimb S1 Are Organized Primarily as Recurrent Loops
}

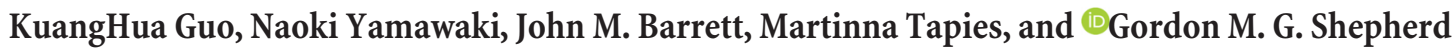 \\ Department of Physiology, Feinberg School of Medicine, Northwestern University, Chicago, Illinois 60611
}

Cortical projections to the thalamus arise from corticothalamic (CT) neurons in layer 6 and pyramidal tract-type (PT) neurons in layer 5B. We dissected the excitatory synaptic connections in the somatosensory thalamus formed by CT and PT neurons of the primary somatosensory (S1) cortex, focusing on mouse forelimb S1. Mice of both sexes were studied. The CT neurons in S1 synaptically excited S1projecting thalamocortical (TC) neurons in subregions of both the ventral posterior lateral and posterior (P0) nuclei, forming a pair of recurrent cortico-thalamo-cortical (C-T-C) loops. The PT neurons in S1 also formed a recurrent loop with S1-projecting TC neurons in the same subregion of the P0. The PT neurons in the adjacent primary motor (M1) cortex formed a separate recurrent loop with M1projecting TC neurons in a nearby subregion of the PO. Collectively, our results reveal that C-T-C circuits of mouse forelimb S1 are primarily organized as multiple cortical cell-type-specific and thalamic subnucleus-specific recurrent loops, with both CT and PT neurons providing the strongest excitatory input to TC neurons that project back to $S 1$. The findings, together with those of related studies of C-T-C circuits, thus suggest that recurrently projecting thalamocortical neurons are the principal targets of cortical excitatory input to the mouse somatosensory and motor thalamus.

Key words: connectivity; channelrhodopsin; corticothalamic; motor; somatosensory; thalamocortical

Significance Statement

Bidirectional cortical communication with the thalamus is considered an important aspect of sensorimotor integration for active touch in the somatosensory system, but the cellular organization of the circuits mediating this process is not well understood. We used an approach combining cell-type-specific anterograde optogenetic excitation with single-cell recordings targeted to retrogradely labeled thalamocortical neurons to dissect these circuits. The findings reveal a consistent pattern: cortical projections to the somatosensory thalamus target thalamocortical neurons that project back to the same cortical area. Commonalities of these findings to previous descriptions of related circuits in other areas suggest that cortico-thalamo-cortical circuits may generally be organized primarily as recurrent loops.

\section{Introduction}

Cortical projections to the thalamus arise from two broad classes of neurons: layer 6 corticothalamic (CT) neurons, which project primarily to the thalamus, and layer $5 \mathrm{~B}$ "pyramidal tract"-type (PT) neurons, which project to multiple subcortical structures, including the thalamus. The growing availability of optogenetic and related tools for circuit analysis enables determination of the cell-type-specific connections from CT and PT axons onto thalamocortical (TC) neurons. Application of such tools to dissect the cortico-thalamo-cortical (C-T-C) circuits of the primary

\footnotetext{
Received Sept. 19, 2019; revised Jan. 29, 2020; accepted Jan. 30, 2020.

Author contributions: K.G., N.Y., J.M.B., and G.M.G.S. designed research; K.G., N.Y., J.M.B., and M.T. performed research; K.G., N.Y., J.M.B., M.T., and G.M.G.S. analyzed data; G.M.G.S. wrote the paper.

Funding was provided by National Institutes of Health Grants MH067564 and NS061963.

The authors declare no competing financial interests.

Correspondence should be addressed to Gordon M. G. Shepherd at g-shepherd@northwestern.edu.

https://doi.org/10.1523/JNEUROSCI.2277-19.2020

Copyright $\odot 2020$ the authors
}

motor (M1) cortex revealed that neither CT nor PT axons strongly excite M1-projecting TC neurons in the ventrolateral (VL) nucleus, but both CT and PT axons strongly excite M1projecting TC neurons in the posterior (PO) nucleus (Yamawaki and Shepherd, 2015). We also recently found that CT and PT axons in a higher-order motor area, the anterolateral motor cortex (ALM), both converge on the ventromedial (VM) nucleus and excite TC neurons that project back to the ALM (Guo et al., 2018). A related pattern was observed in the prefrontal cortex (PFC), where both CT and PT axons converged on and excited PFC-projecting TC neurons, in this case located in both the mediodorsal (MD) and VM nuclei (Collins et al., 2018). Although the C-T-C circuits of each area differ in some details, a common feature appears to be the targeting of recurrently projecting TC neurons in matrix-type thalamic nuclei (VM, PO). This pattern could be a specialization associated with higher-order frontal areas and systems (Collins and Anastasiades, 2019). However, a similar pattern is seen for the thalamic connections of the fore- 
limb area of the M1, with both CT and PT axons exciting recurrently projecting TC neurons in both the VM and PO nucleus (Yamawaki and Shepherd, 2015; Guo et al., 2018). Anatomical studies suggest that a similar pattern could pertain to the $\mathrm{C}-\mathrm{T}-\mathrm{C}$ circuits of the primary somatosensory (S1) cortex, as both CT and PT axons from the whisker-related (barrel) S1 cortex project to the PO nucleus (Deschênes et al., 1994, 1998; Bourassa et al., 1995; Killackey and Sherman, 2003; Chevée et al., 2018; Hoerder-Suabedissen et al., 2018). The extent to which these projections innervate $\mathrm{S} 1$-projecting TC neurons in the PO to form recurrent loops has not been determined.

To further explore C-T-C loops, and as part of a broader effort to elucidate the circuit organization of ascending pathways conveying forelimb somatosensory signals via the thalamus and $\mathrm{S} 1$ to $\mathrm{M} 1$, we dissected the thalamic connections of the CT and PT neurons of forelimb S1 in the mouse. To do so, we used a strategy of combining electrophysiological recordings from retrogradely labeled thalamic neurons and optogenetic photostimulation of selectively labeled axons (Yamawaki and Shepherd, 2015; Yamawaki et al., 2016b; Guo et al., 2018). The results delineate multiple celltype-specific connections that consistently involve preferential targeting of recurrently projecting TC neurons, implying that recurrent loops undergird the circuit architecture of both the CT and PT components of forelimb somatosensory C-T-C circuits.

\section{Materials and Methods}

Animals. Animal studies followed the guidelines of the National Institutes of Health and the Society for Neuroscience and were approved by the Northwestern University Animal Care and Use Committee. The following mouse strains were used in these studies, maintained as in-house breeding colonies. In the main set of experiments, for cell-type-specific expression we used the Cre lines retinol binding protein 4 (Rbp4)-Cre [Rbp4_KL100-Cre, Mutant Mouse Resource and Research Center (MMRRC); Gerfen et al., 2013] and neurotensin receptor 1 (Ntsr1)-Cre (Ntsr1_GN220-Cre, MMRRC; Gong et al., 2007), each backcrossed with C57BL/6 mice for at least six generations. In one anatomical experiment, we crossed sodium channel epithelial 1 subunit alpha (Scnnla)-Cre mice (Scnn1a-Tg3-Cre, JAX 009613, The Jackson Laboratory; Madisen et al., 2010), which express Cre in cortical layer 4 neurons, with a Credependent nuclear-targeted mCherry (nt-mCherry) reporter line (Rosa26LSL-H2B-mCherry, JAX 023139, The Jackson Laboratory; Peron et al., 2015); in another, we used glutamate decarboxylase 2 (Gad2)-mCherry mice (Gad2-T2a-NLS-mCherry, JAX 023140, The Jackson Laboratory; Peron et al., 2015). Mice of both sexes were used; analysis of the datasets after disaggregating by sex did not show any significant sex-dependent differences, and so the data were kept pooled. Animals were housed with a $12 \mathrm{~h}$ light/dark cycle, with ad libitum access to water and food. Mice were 1.5-3 months old at the time of the initial surgery and were used for experiments 3-6 weeks later. Animal numbers for each type of experiment are given in the text and figures.

Labeling. Stereotaxic injections were performed as described previously (Yamawaki and Shepherd, 2015; Guo et al., 2018). In brief, mice were deeply anesthetized with isoflurane and placed in a stereotaxic frame. Ophthalmic ointment was applied to protect the eyes during sur- gery. Thermal support was provided using a feedback-controlled heating pad (Warner Instruments). Mice were given preoperative analgesic coverage $(0.3 \mathrm{mg} / \mathrm{kg}$ buprenorphine subcutaneously). Small craniotomies were opened directly over cortical targets in the right hemisphere. Injection pipettes were fabricated from glass capillary micropipettes (Wiretrol II, Drummond Scientific), pulled (PP-830, Narishige) to a fine tip, and beveled (microgrinder EG-400, Narishige) to a sharp edge. Pipettes were loaded with virus or retrograde tracers (red RetroBeads, Lumafluor; or, cholera toxin subunit B conjugated with AlexaFluor 647, CTB647, Life Technologies) by tip filling (i.e., application of negative pressure to the back of the pipette). Pipettes were advanced slowly to their targets, where $40-100 \mathrm{nl}$ of virus was injected, and kept in place for several minutes before retraction. Injections of virus and tracer at a single cortical site were made by sequentially inserting the pipettes at nominally identical coordinates. Animals received postoperative analgesic coverage $(1.5 \mathrm{mg} / \mathrm{kg}$ meloxicam subcutaneously once every $24 \mathrm{~h}$ for $2 \mathrm{~d}$ ) and were maintained for at least 3 weeks before slice experiments. Experiment-specific details are as follows.

In experiments examining cortical input to thalamic neurons, we used Rbp4-Cre and Ntsr1-Cre mice to selectively label the PT or CT components, respectively, that together comprise the cortical projection to the thalamus. Previous studies have characterized the cellular specificity of Cre expression in cortical neurons in these lines (Gong et al., 2007; Gerfen et al., 2013; Bortone et al., 2014; Yamawaki and Shepherd, 2015; Guo et al., 2018; multiple transgenic characterizations available at http:// connectivity.brain-map.org/transgenic, e.g., \#167642756 for Rbp4-Cre) and have used these lines for selectively labeling the PT and CT components of the cortical projection to the thalamus (Kim et al., 2014; Yamawaki and Shepherd, 2015; Grant et al., 2016; Jeong et al., 2016; Guo et al., 2018; Harris et al., 2019); a general caveat is that, although these lines give highly specific and robust labeling, it is possible that they miss certain subtypes. Specifically, Cre-dependent adeno-associated virus (AAV)channelrhodopsin-2 (ChR2; AAV5.Ef1a.DIO.hChR2(E123T/T159C)EYFP.WPRE.hGH, 35509, Addgene) was injected into either the forelimb S1 or forelimb M1 of either Ntsr1-Cre mice (to label layer 6 CT neurons) or Rbp4-Cre mice (to label layer 5 intratelencephalic and PT neurons). Both the $\mathrm{S} 1$ and $\mathrm{M} 1$ were also injected with retrograde tracer to 
A
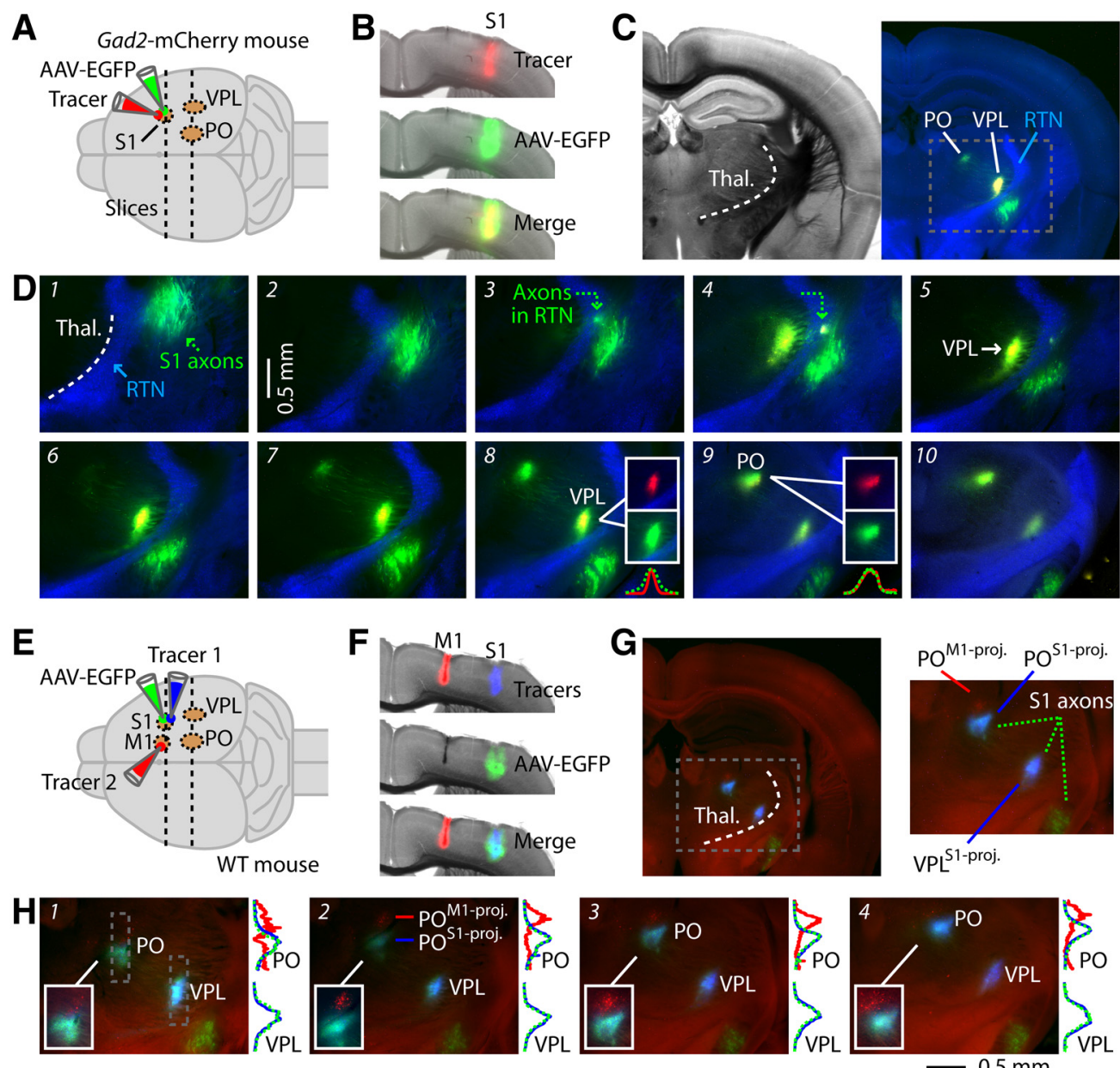

-..S1 axons - S1-projecting -M1-projecting
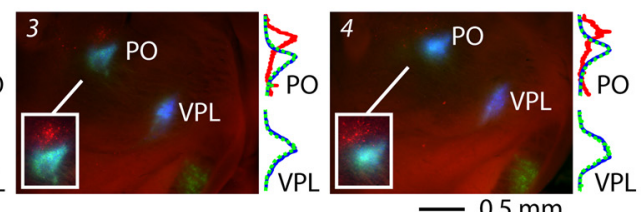

Figure 2. Anatomy of forelimb S1-related C-T-C pathways in thalamus. A, Schematic of injection strategy: both AAV-EGFP and CTB647 were injected into the forelimb S1 area of a Gad2-mCherry mouse. B, Bright-field/fluorescence images of a coronal slice containing the cortical injection site, showing the red (top) and green (middle) channels separately and merged (bottom). $\boldsymbol{C}$, Bright-field (left) and merged fluorescence (right) images of a coronal slice at the level of the VPL and PO nuclei of the thalamus. The thalamic RTN is labeled with Gad2-mCherry signal (for clarity, displayed as blue). D, Higher-magnification views of the labeling pattern across the thalamus (series of $150 \mu \mathrm{m}$ slices). The RTN is labeled with Gad2-mCherry signal (blue). Axons from S1 (green) pierce the RTN anteriorly and then course posteriorly, ramifying in subregions of the VPL and PO nuclei. Insets show the red and green channels separately, and their horizontal fluorescence profiles (bottom traces; peak normalized), for the labeling patterns in VPL (8) and PO (9). $\boldsymbol{E}$, Schematic of injection strategy: in a WT mouse, both AAV-EGFP and (TB647 were injected into the forelimb S1 area, and a second retrograde tracer (red RetroBeads) was injected into the forelimb M1 area. $\boldsymbol{F}$, The cortical injection sites (coronal slice). G, Merged fluorescence image (coronal slice) at the level of the VPL and P0 nuclei of the thalamus, with the thalamic region of interest shown enlarged (right) and labeled to indicate the S1 axons (green) and the S1-projecting (blue) and M1-projecting (red) TC neurons (cyan: overlap of green and blue labeling). $\boldsymbol{H}$, Higher-magnification views of the labeling pattern across the thalamus (series of $150 \mu \mathrm{m}$ slices). Insets: enlarged views of P0 labeling (contrast and brightness optimized). Plots: vertical fluorescence profiles (peak normalized) of labeling in PO (top; with all three channels) and VPL (bottom; green and blue channels only, as there was no labeling in the red channel).

label thalamocortical neurons in the ventral posterior lateral (VPL) and PO nuclei. The S1 coordinates (relative to the bregma) were as follows: $0.0-0.7 \mathrm{~mm}$ posterior, $2.2-2.5 \mathrm{~mm}$ lateral, $0.1-1 \mathrm{~mm}$ deep. The M1 coordinates (relative to the bregma) were as follows: $0.5-0.0 \mathrm{~mm}$ anterior, 1.1-1.4 mm lateral, 0.1-1 $\mathrm{mm}$ deep.

Circuit analysis. Brain slices were prepared as described previously (Guo et al., 2018). In brief, mice that had undergone in vivo labeling were killed by anesthetic overdose and decapitation. Brains were rapidly removed and placed in chilled cutting solution (composition, in mM: 110 choline chloride, 11.6 sodium L-ascorbate, 3.1 pyruvic acid, $25 \mathrm{NaHCO}_{3}$, 25 D-glucose, $2.5 \mathrm{KCl}, 7 \mathrm{MgCl}_{2}, 0.5 \mathrm{CaCl}_{2}, 1.25 \mathrm{NaH}_{2} \mathrm{PO}_{4}$, aerated with $95 \% \mathrm{O}_{2} / 5 \% \mathrm{CO}_{2}$ ). Brains were trimmed using blocking cuts angled to yield coronal slices $(0.25 \mathrm{~mm})$. Slices were cut (VT1200S, Leica) in chilled cutting solution and transferred to artificial CSF (ACSF) composed of the following (in mM): $127 \mathrm{NaCl}, 25 \mathrm{NaHCO}_{3}, 25$ D-glucose, $2.5 \mathrm{KCl}, 1$ $\mathrm{MgCl}_{2}, 2 \mathrm{CaCl}_{2}, 1.25 \mathrm{NaH}_{2} \mathrm{PO}_{3}$. Slices were incubated at $34^{\circ} \mathrm{C}$ for $30 \mathrm{~min}$ and then kept at $22^{\circ} \mathrm{C}$ for at least an hour before recording.

Brain slices were transferred to the recording chamber of an upright microscope (BX51WI chassis, Olympus) equipped for whole-cell elec- trophysiology and photostimulation, as previously described (Guo et al., 2018). The bath solution consisted of ACSF warmed to $34^{\circ} \mathrm{C}$ by an in-line feedback-controlled heater (TC 324B, Warner Instruments). Patch pipettes were fabricated by pulling (P-97, Sutter Instrument) borosilicate glass (inner diameter 0.86, outer diameter $1.5 \mathrm{~mm}$, with filament; Warner Instruments) to a fine tip (resistance of 2-4 M $\Omega$ ). Recordings were made in voltage-clamp or current-clamp mode using pipettes filled with cesium-based or potassium-based internal solution (composition: 128 mM cesium or potassium methanesulfonate, $10 \mathrm{~mm}$ HEPES, $10 \mathrm{~mm}$ phosphocreatine, $4 \mathrm{~mm} \mathrm{MgCl}_{2}$, 4 mм ATP, $0.4 \mathrm{~mm}$ GTP, 3 mm ascorbate, $1 \mathrm{~mm}$ EGTA, $1 \mathrm{~mm}$ QX-314, $0.05 \mathrm{~mm}$ Alexa Fluor hydrazide, $4 \mathrm{mg} / \mathrm{ml}$ biocytin, pH 7.25, 290-295 mOsm).

A video camera (Retiga 2000R, QImaging) was used to image slices and cells under wide-field gradient-contrast or epifluorescence optics. Labeling patterns of retrogradely labeled somata and anterogradely labeled axons were visualized using LED illumination (M470L2, M530L2, M660L3, Thorlabs) and standard filter sets (U-N41017, U-N31002, Chroma, CY5-4040C-OMF, Semrock). Pipettes were advanced under positive pressure to establish $>1 \mathrm{G} \Omega$ seals onto retrogradely labeled neu- 

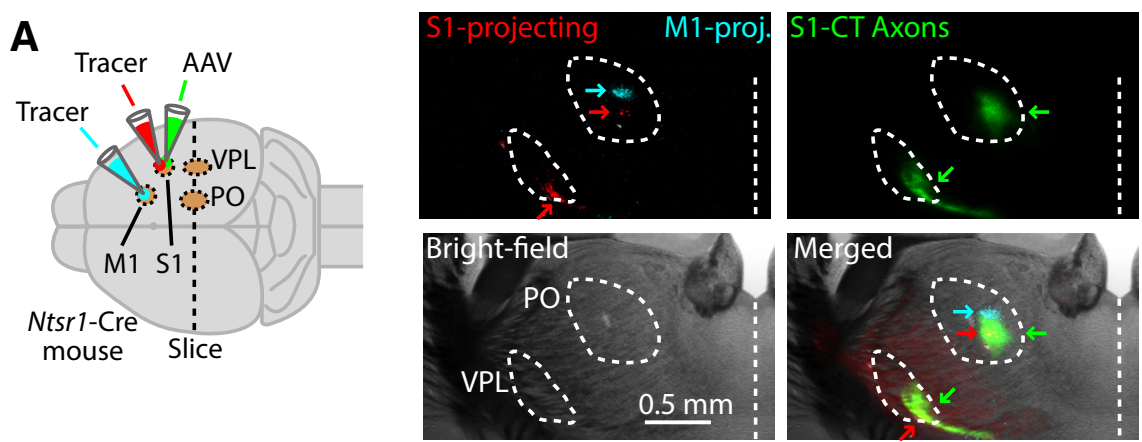

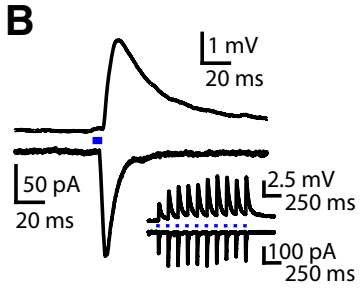

E

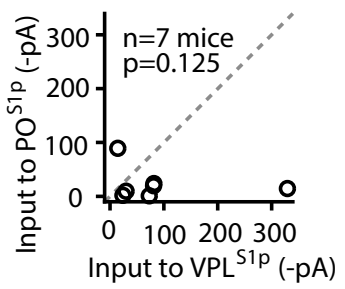

C

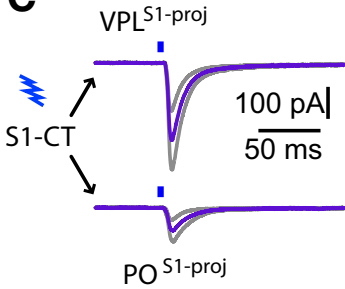

$\mathbf{F}$

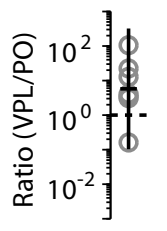

D

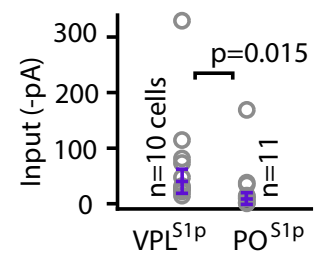

G

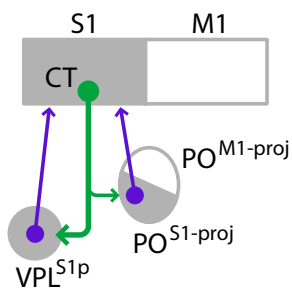

Figure 3. S1-CT axons excite S1-projecting TC neurons in the VPL and PO.A, Schematic (left) of injections of retrograde tracers in S1 and M1, and Cre-dependent AAV-ChR2 in M1, of an Ntsr1-Cre mouse. Images (right) are from a coronal slice through the somatosensory nuclei of the thalamus showing retrograde labeling of S1-projecting and M1-projecting neurons (top left), anterograde labeling of axons (top right), and bright-field (bottom left) and merged (bottom right) images. $\boldsymbol{B}$, Example traces showing excitatory synaptic responses (top, EPSP; bottom, EPSC) recorded in a VPL ${ }^{51-p r o j}$ neuron, evoked by photostimulation of ChR2-expressing S1-CT axons. Inset: responses to trains of stimuli. C, EPSCs recorded in retrogradely labeled TC neurons. Single photostimuli (blue bars above traces) were used to activate ChR2-expressing axons. Traces represent group-averaged responses ( \pm SEM; gray lines). D. Cell-based group comparison of input to individual neurons (circles). Error bars represent the median input across cells \pm m.a.d. The $p$ value for the rank-sum test comparing the two groups is shown, along with the numbers of cells per group. $\boldsymbol{E}$, Animal-based group comparison of input recorded in the two cell types, averaged for each animal (circles). The $p$ value for the sign test comparing the two groups is shown along with the number of animals. $\boldsymbol{F}$, Animal-based ratios of input to the two cell types, averaged for each animal (circles), along with the geometric mean and the geometric standard factor (bars). G, Schematic depiction of the cellular connectivity pattern.

rons. After membrane rupture to establish whole-cell configuration, intracellular recordings were made with the amplifier (MultiClamp 700B, Molecular Devices) in voltage-clamp mode. Recordings with series resistance $>40 \mathrm{M} \Omega$ were excluded. Ephus software (www.ephus.org; Suter et al., 2010) was used for hardware control and data acquisition.

For wide-field photostimulation, as previously described (Guo et al., 2018), a blue LED (M470L2, Thorlabs) was driven with a TTL (transistor-transistor logic) pulse to generate photostimuli with a duration of 5 $\mathrm{ms}$. The LED intensity controller was set to deliver $1 \mathrm{~mW} / \mathrm{mm}^{2}$ at the level of the specimen. For each cell, photostimulation trials were repeated three times at an interstimulus interval of $30 \mathrm{~s}$ while recording in voltageclamp mode with the command potential set to $-70 \mathrm{mV}$ to isolate EPSCs. We did not pharmacologically block inhibition, as the command potential was near the GABAergic reversal potential and recordings were generally in slices in which axons emanating from the S1-recipient region of the thalamic reticular nucleus (RTN) were severed.

To quantify evoked synaptic responses, for each cell the traces from several (generally three) trial repetitions were averaged, and the response amplitude was calculated as the mean over a poststimulus interval of 50 $\mathrm{ms}$. Data were compared by pooling across slices and animals, as in previous studies (Guo et al., 2018). Pairwise comparisons were made using the absolute or normalized response amplitudes, as indicated in the text.

Flavoprotein autofluorescence imaging. Methods for in vivo transcranial flavoprotein autofluorescence imaging (Shibuki et al., 2003) were gener- ally as described previously for auditory cortex mapping (Joshi et al., 2015), adapted for somatosensory cortex mapping. In brief, WT mice first underwent a surgical procedure to mount a head-post as previously described (Li et al., 2018). Mice received $0.3 \mathrm{mg} / \mathrm{kg}$ buprenorphine preoperatively and $1.5 \mathrm{mg} / \mathrm{kg}$ meloxicam postoperatively as analgesia, followed by a second dose of meloxicam $24 \mathrm{~h}$ after surgery. Mice recovered $\geq 24 \mathrm{~h}$ before imaging. To image flavoprotein autofluorescence responses, head-post-mounted mice were intraperitoneally injected with $80-100 \mathrm{mg} / \mathrm{kg}$ ketamine and 5-15 mg/kg xylazine to induce anesthesia. During imaging, mice were thermally supported and given maintenance doses of ketamine-xylazine as needed. Tactile stimulation was performed on head-fixed mice using a $40 \times 10 \times 0.5 \mathrm{~mm}$ piezoelectric bimorph element (SMBA4510T05M, Steiner \& Martins) bearing a thin plastic probe, driven by a linear amplifier (EPA-007-012, Piezo Systems) with a $1 \mathrm{~s}$ duration, $20 \mathrm{~V}, 20 \mathrm{~Hz}$ sinusoidal signal that generated a peak-to-peak displacement of $\sim 1 \mathrm{~mm}$ at the probe tip. The probe was positioned against the forepaw or hindpaw, or within the array of whiskers. On each trial, the skull was illuminated for $5 \mathrm{~s}$ with a blue LED (M470L2, Thorlabs) at maximum intensity. Stimulation was delivered on alternate trials $3 \mathrm{~s}$ after the onset of illumination. The LED was turned off for $3 \mathrm{~s}$ between trials to allow flavoprotein autofluorescence to recover. Images were acquired using a monochrome camera (BFS-U3-32S4M-C, FLIR Systems) at a frame rate of 20 frames/s with $40 \mathrm{~ms}$ exposure, $17.9 \mathrm{~dB}$ gain, and $2 \times$ 2 binning and recorded using SpinView (FLIR Systems) as uncom- 
A

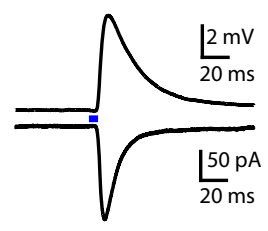

B
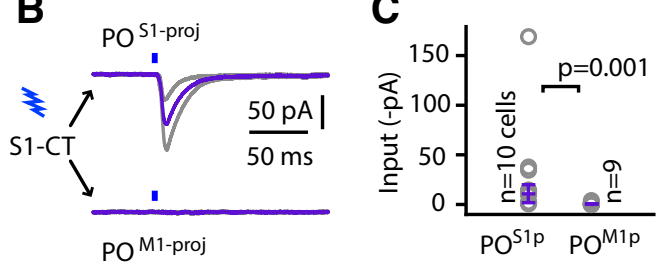

$\mathbf{E}$

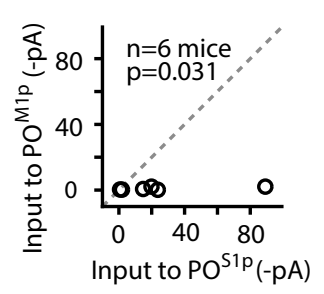

$\mathbf{F}$

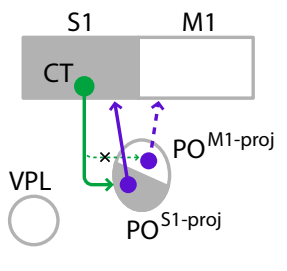

Figure 4. S1-CT input is stronger to S1-projecting than M1-projecting P0 neurons. $A$, Example traces showing excitatory synaptic responses (top, EPSP; bottom, EPSC) recorded in a $\mathrm{PO}^{\text {S1-proj }}$ neuron, evoked by photostimulation of ChR2-expressing S1-CT axons. B, EPSCs recorded in retrogradely labeled TC neurons. Single photostimuli (blue bars above traces) were used to activate ChR2-expressing axons. Traces represent group-averaged responses ( \pm SEM; gray lines). C, Cell-based group comparison of input to individual neurons (circles). Error bars represent the median input across cells \pm m.a.d. The $p$ value for the rank-sum test comparing the two groups is shown, along with the numbers of cells per group. $D$, Animal-based group comparison of input recorded in the two cell types, averaged for each animal (circles). The $p$ value for the sign test comparing the two groups is shown, along with the number of animals. $E$, Animal-based ratios of input to the two cell types, averaged for each animal (circles), along with the geometric mean and the geometric standard factor (bars). $\boldsymbol{F}$, Schematic depiction of the cellular connectivity pattern.

pressed video files. Stimulus delivery and image acquisition were controlled and synchronized using WaveSurfer (wavesurfer.janelia.org) and an NI USB-6229 data acquisition board (National Instruments). Images were imported into MATLAB (MathWorks) and further binned into $16 \times 16$ pixel bins. The $\Delta F / F_{0}$ maps of cortical responses for each animal and body part were calculated by subtracting out and dividing by the average fluorescence image during the $2 \mathrm{~s}$ before stimulation on each trial, then averaging over trials. Bright-field images were also collected and used to identify the bregma, to align and average maps across animals. For presentation of maps for each body part separately, $\Delta F / F_{0}$ maps were thresholded at zero and peak normalized. For combined body part maps, $\Delta F / F_{0}$ values were normalized identically across body parts to lie within the range $[0,1]$, and the map for each body part was assigned to a different color channel.

Experimental design and statistical analysis. Group comparisons were made using nonparametric tests as indicated in the text, with significance defined as $p<0.05$. Unpaired data were compared using the rank-sum test. Paired data were compared using the sign test. For group data, medians and median average deviations (m.a.d.) were calculated as descriptive statistical measures of central tendency and dispersion, except for ratios, for which geometric means and standard factors were calculated. Statistical analyses were conducted using standard MATLAB functions.

\section{Results}

S1-CT axons excite S1-projecting TC neurons in VPL and PO As a preliminary step to dissecting the C-T-C circuits of forelimb $\mathrm{S} 1$, we confirmed its anatomical localization. First, consistent with the location of forelimb S1 in the reference atlas of the Allen Mouse Brain Atlas (Lein et al., 2007), we observed layer 4 labeling at this stereotaxic location in a mouse line (Scnnla-Cre) that labels this layer in the somatosensory cortex, including the forelimb representation (Fig. 1A). Second, consistent with the thalamocortical pathway organization in the somatosensory system, stereotaxic injection of tracer into this forelimb S1 site resulted in the expected pattern of retrograde labeling within the VPL nucleus of the thalamus, the primary somatosensory nucleus in forelimb-related thalamocortical pathways, with additional la- beling in the PO nucleus (Fig. 1B). Third, consistent with these anatomical localizations, functional mapping in wild-type mice using flavoprotein autofluorescence imaging (see Materials and Methods) showed that cortical responses to tactile stimulation of the forepaw were also localized to this area (Fig. 1C), similar to previous findings (Ferezou et al., 2007; Auffret et al., 2018).

We also used anterograde and retrograde labeling to gain an overview of the thalamic anatomy of C-T-C pathways of forelimb S1. We injected the forelimb S1 area with both Cre-independent AAVEGFP, which anterogradely labeled the combined CT and PT components of the cortical projection to the thalamus, and retrograde tracer (CTB647), which labeled the cortically projecting thalamic neurons. In one case (Fig. $2 A-C$ ), we used a Gad2-mCherry mouse, which facilitated visualization of the RTN as well; similar results were also observed with WT mice $(n=4)$. In a series of slices spanning the thalamus (Fig. 2D), S1 axons were observed to traverse the RTN at relatively anterior levels before ramifying more posteriorly in subregions of the VPL and PO nuclei. Within both nuclei, the labeling patterns of the $\mathrm{S} 1$ axons closely matched and overlapped with those of the S1-projecting TC neurons. In the experiments with the four WT mice, as shown in a representative example (Fig. $2 E-G$ ), we used the same injection scheme and, for comparison, additionally injected a second tracer into the nearby forelimb M1 area as a way to visualize the distributions of both S1-projecting and M1-projecting TC neurons in relation to the S1 axons. Inspection of a series of thalamic slices showed (Fig. $2 \mathrm{H}$ ) that a subregion of the VPL nucleus was labeled by retrograde tracer injected into $S 1$ but not $\mathrm{M} 1$, and that $\mathrm{S} 1$ axons overlapped with S1-projecting VPL neurons (VPL ${ }^{\text {S1-proj }}$ ). The PO was labeled by retrograde tracers injected into both $\mathrm{S} 1$ and $\mathrm{M} 1$ but in largely nonoverlapping clusters, with the S1-projecting PO neurons $\left(\mathrm{PO}^{\mathrm{S1-proj}}\right)$ located just ventral and lateral to $\mathrm{PO}^{\mathrm{M1} \text {-proj }}$ neurons. The S1 axons generally overlapped extensively with the clusters of $\mathrm{PO}^{\mathrm{S1-proj}}$ neurons and minimally with the labeled $\mathrm{PO}^{\mathrm{M} 1-\text { proj }}$ neurons.

To dissect the C-T-C circuits of forelimb S1 at the level of cell-type-specific excitatory synaptic connectivity, we first focused on the thalamic connections of the CT neurons. Previous studies, mainly in whisker-related pathways, have shown that S1-CT neurons project mainly to the ventral posterior medial nucleus but also to the PO nucleus (Bourassa et al., 1995; Killackey and Sherman, 2003; Chevée et al., 2018; HoerderSuabedissen et al., 2018). Therefore, we first assessed the relative strength of connections from S1-CT neurons to thalamic neurons located in the VPL and PO. Because the PO contains TC neurons with various projections, not only to $\mathrm{S} 1$ but also M1 (Deschênes et al., 1998; Ohno et al., 2012; Hooks et al., 2013; Yamawaki and Shepherd, 2015), we targeted our recordings in the PO to TC neurons identified by retrograde labeling as projecting back to $\mathrm{S} 1$ or to the adjacent M1 cortex. We followed a strategy of photostimulating ChR2-expressing S1-CT axons while recording from S1-projecting or M1-projecting TC neu- 
rons in slices of thalamus. For this, similar to the injection scheme for the anatomical experiment described earlier, we injected AAV-DIO-ChR2-YFP in S1 of Ntsr1-Cre mice to label S1-CT neurons and their axons with ChR2, and injected retrograde tracers (different colors) into S1 and M1 (Fig. $3 A$, left). In slices of the thalamus (Fig. $3 A$, right), we observed the same pattern of labeling as described earlier (i.e., S1 axons colocalized with $\mathrm{VPL}^{\text {S1-proj }}$ and $\mathrm{PO}^{\mathrm{S1} \text {-proj }}$ neurons in subregions of those nuclei, with $\mathrm{PO}^{\mathrm{M} 1 \text {-proj }}$ neurons located in a suprajacent subregion of the PO).

With this labeling approach, we targeted whole-cell recordings to retrogradely labeled VPL ${ }^{\mathrm{S} 1-\text { proj }}$ and $\mathrm{PO}^{\mathrm{S1-proj}}$ neurons and sampled excitatory synaptic responses evoked by photostimulating S1-CT axons. The recorded neurons were generally located in the midst of the highdensity region of the cluster of labeled neurons rather than near the margins. As shown in the example traces from a VPL ${ }^{\text {S1-proj }}$ neuron (Fig. 3B), recordings showed large EPSCs and EPSPs, with short-term facilitation as expected for somatosensory CT $\rightarrow$ TC synapses (Crandall et al., 2015). For quantification of input strength, we recorded in voltage-clamp mode and measured the amplitudes of evoked EPSCs (Fig. 3C). In this set of experiments ( $n=7$ Ntsr1-Cre mice), evoked EPSC input from S1-CT axons to $\mathrm{VPL}^{\text {S1-proj }}$ neurons was relatively strong (median across 10 cells, $39.5 \pm 21.5 \mathrm{pA}$; median for 7 mice, $73.1 \pm 43.4 \mathrm{pA}$ ). Input to $\mathrm{PO}^{\text {S1-proj }}$ neurons was also substantial (median across 11 cells, $9.6 \pm 7.7 \mathrm{pA}$; median for 7 mice, $14.7 \pm 8.9 \mathrm{pA}$ ), albeit weaker based on pooling all cells (unpaired cell-based comparison, $p=0.015$, rank-sum test; Fig. 3C), although not when averaging per animal (pairwise animal-based comparison, $p=0.125$, sign

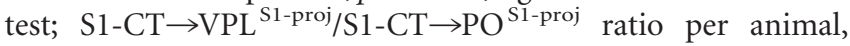
$5.76 \pm 7.54$, geometric mean \pm geometric standard factor; Fig. $3 E, F)$.

These results show that the axonal projections of S1-CT neurons to the thalamus are focused on subregions of the VPL and PO. These subregions coincide anatomically with where S1-projecting TC neurons are most densely labeled, and S1-CT axons provide substantial excitatory synaptic input to S1-projecting TC neurons in both the VPL and PO, with somewhat stronger input to those in the VPL. These results thus delineate two cell-type-specific recurrent circuits, one formed by $\mathrm{S} 1-\mathrm{CT} \rightarrow \mathrm{VPL}^{\mathrm{S} 1-\text { proj }}$ connections and the other by $\mathrm{S} 1-\mathrm{CT} \rightarrow \mathrm{PO}^{\text {S1-proj }}$ connections (Fig. 3G).

\section{S1-CT input is stronger to S1-projecting than M1-projecting PO neurons}

Next, we investigated the extent to which the same S1-CT projections that branch to the $\mathrm{PO}$ nucleus also provide excitation to TC
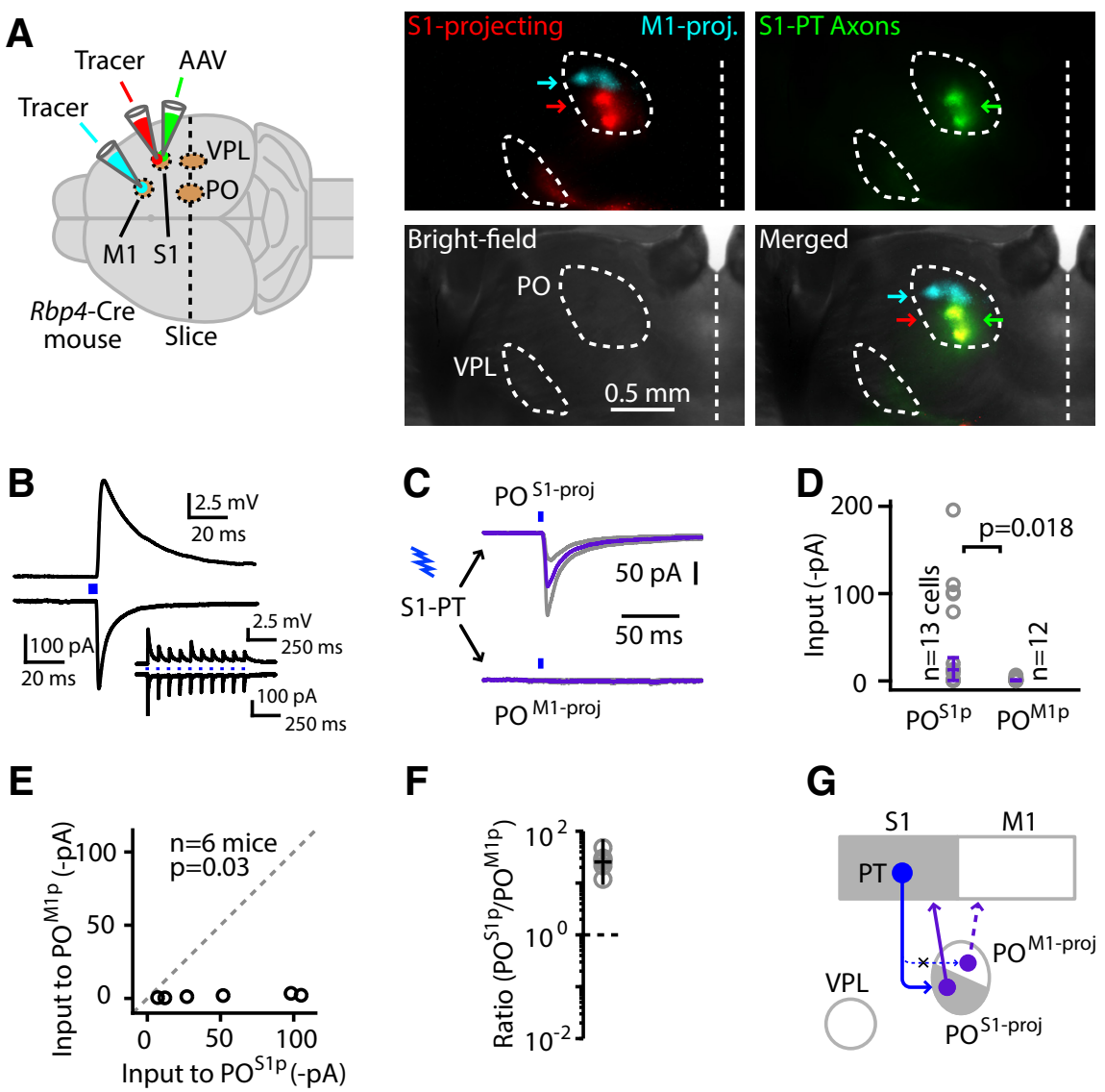

Figure 5. S1-PT input is stronger to S1-projecting than M1-projecting P0 neurons. $\boldsymbol{A}$, Schematic (left) of injections of retrograde tracers in S1 and M1, and Cre-dependent AAV-ChR2 in S1, of an Rbp4-Cre mouse. Images (right) are from a onal slice through the somatosensory nuclei of the thalamus showing retrograde labeling of S1-projecting and M1( images. $\boldsymbol{B}$, Example traces showing excitatory synaptic responses (top, EPSP; bottom, EPSC) recorded in a PO ${ }^{51-p r o j}$

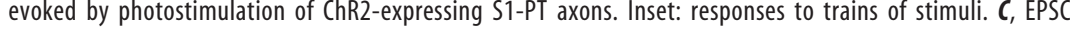
expressing axons. Traces represent group-averaged responses ( \pm SEM; gray lines). D. Cell-based group comparison of rank-sum test comparing the two groups is shown, along with the numbers of cells per group. $\boldsymbol{E}$, Animal-based group comparison of input recorded in the two cell types, averaged for each animal (circles). The $p$ value for the sign test 列 Schematic depiction of the cellular connectivity pattern.

neurons projecting to $\mathrm{M} 1$, in addition to those projecting to $\mathrm{S} 1$. For this, we used the same injection scheme in Ntsr1-Cre mice as described earlier (Fig. $3 A, B$ ) but focused on recording from $\mathrm{PO}^{\mathrm{S} 1 \text {-proj }}$ and $\mathrm{PO}^{\mathrm{M} 1 \text {-proj }}$ neurons (Fig. $4 A, B$ ). In this set of experiments ( $n=6$ Ntsr1-Cre mice, including most of the same mice used in the previous experiment), S1-CT input to $\mathrm{PO}^{\mathrm{S1}-\text { proj }}$ neurons was again substantial (median across 10 cells, $11.1 \pm 9.3 \mathrm{pA}$; median for $n=6$ mice, $17.3 \pm 10.9 \mathrm{pA}$ ), but S1-CT input to $\mathrm{PO}^{\mathrm{M1} \text {-proj }}$ neurons was extremely weak (median across nine cells, $0.3 \pm 0.3 \mathrm{pA}$; median for $n=6$ mice, $0.5 \pm 0.3 \mathrm{pA}$ ), a statistically significant difference in cell-based and animal-based comparisons (unpaired cell-based comparison, $p=0.001$, rank-sum test; pairwise animal-based comparison, $p=0.031$, sign test; S1-CT to $\mathrm{PO}^{\mathrm{S} 1 \text {-proj }} / \mathrm{PO}^{\mathrm{M} 1 \text {-proj }}$ ratio per animal, $16.84 \pm 4.46$, geometric mean \pm geometric standard factor; Fig. $4 C-E$ ).

These results indicate that S1-CT neurons provide much stronger input to $\mathrm{PO}^{\mathrm{S1-proj}}$ neurons than to $\mathrm{PO}^{\mathrm{M1-proj}}$ neurons. These electrophysiological findings accord with the anatomical 

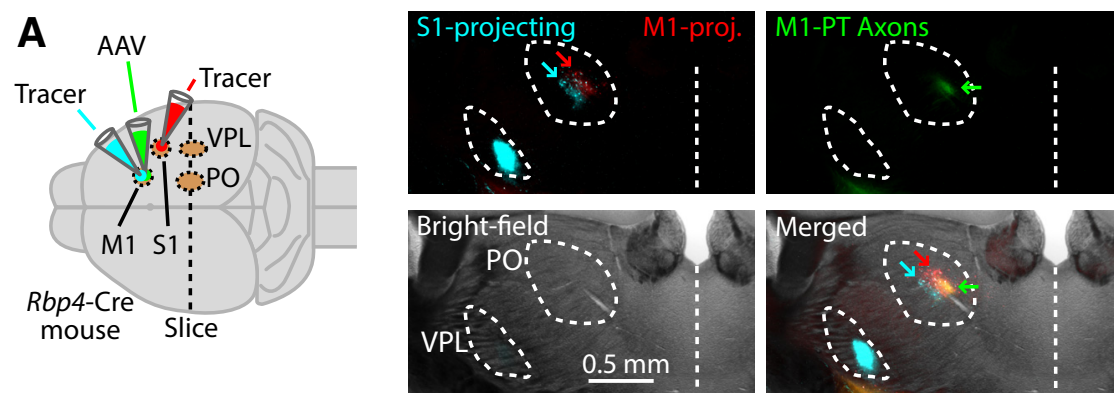

B
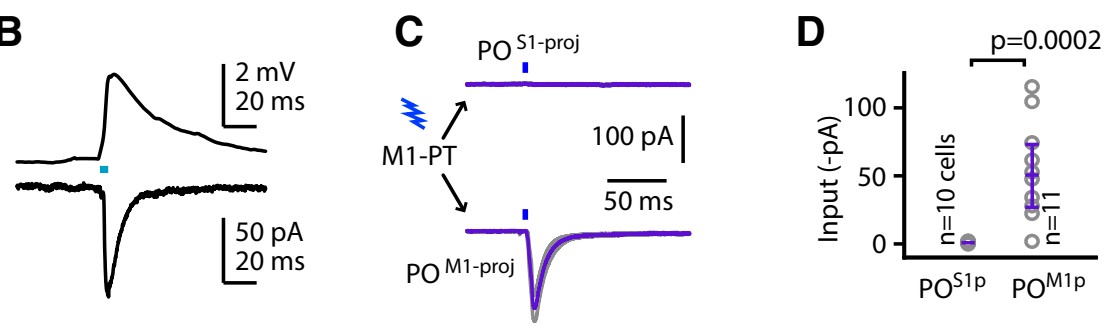

E

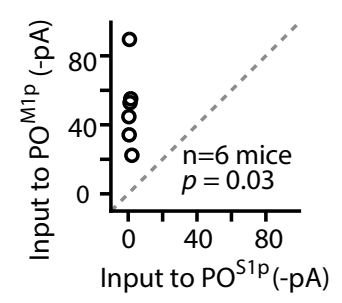

$\mathbf{F}$

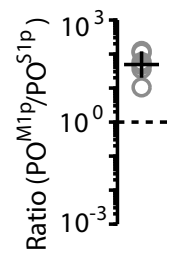

G

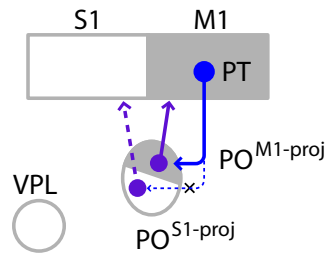

Figure 6. M1-PT input is stronger to M1-projecting than S1-projecting P0 neurons. (A) Schematic (left) of injections of retrograde tracers in S1 and M1, and Cre-dependent AAV-ChR2 in M1, of an Rbp4-Cre mouse. Images (right) are from a coronal slice through the somatosensory nuclei of the thalamus showing retrograde labeling of S1-projecting and M1-projecting neurons (top left), anterograde labeling of axons (top right), and bright-field (bottom left) and merged (bottom right) images. $\boldsymbol{B}$, Example traces showing excitatory synaptic responses (top, EPSP; bottom, EPSC) recorded in a PO ${ }^{\text {M1-proj }}$ neuron, evoked by photostimulation of ChR2-expressing M1-PT axons. C, EPSCs recorded in retrogradely labeled TC neurons. Single photostimuli (blue bars above traces) were used to activate ChR2-expressing axons. Traces represent group-averaged responses ( \pm SEM; gray lines). D, Cell-based group comparison of input to individual neurons (circles). Error bars represent the median input across cells \pm m.a.d. The $p$ value for the rank-sum test comparing the two groups is shown, along with the numbers of cells per group. $\boldsymbol{E}$, Animal-based group comparison of input recorded in the two cell types, averaged for each animal (circles). The $p$ value for the sign test comparing the two groups is shown, along with the number of animals. $\boldsymbol{F}$, Animal-based ratios of input to the two cell types, averaged for each animal (circles), along with the geometric mean and the geometric standard factor (bars). G, Schematic depiction of the cellular connectivity pattern.

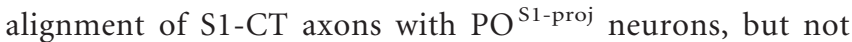
$\mathrm{PO}^{\mathrm{M} 1-\text { proj }}$ neurons. The findings suggest that the C-T-C circuit formed by S1-CT inputs to PO is primarily configured as a closed (i.e., $\mathrm{S} 1 \rightarrow \mathrm{PO} \rightarrow \mathrm{S} 1$ ) rather than open (i.e., $\mathrm{S} 1 \rightarrow \mathrm{PO} \rightarrow \mathrm{M} 1$ ) loop (Fig. $4 F)$.

\section{S1-PT input is stronger to S1-projecting than M1-projecting PO neurons}

Because the axonal projections of S1-PT neurons can send branches to the thalamus, and particularly to the PO (Deschênes et al., 1994, 1998), we next investigated the C-T-C circuits of S1-PT neurons. To dissect the cell-type-specific connections in these circuits, we photostimulated ChR2-expressing S1-PT axons in thalamic brain slices while recording from TC neurons in PO identified as projecting to S1 or M1. We injected AAV-DIOChR2-YFP in S1 of Rbp4-Cre animals to express ChR2 in S1-PT neurons and axons, and injected two different colored tracers into $\mathrm{S} 1$ and $\mathrm{M} 1$ (Fig. 5A, left). Again, $\mathrm{PO}^{\mathrm{S1-proj}}$ neurons were found somewhat ventral and lateral to $\mathrm{PO}^{\mathrm{M1} \text {-proj }}$ neurons (Fig.
$5 A$, right). Similar to the S1-CT axons, the S1-PT axons overlapped extensively with $\mathrm{PO}^{\text {S1-proj }}$ neurons. With this labeling approach, we targeted whole-cell recordings to retrogradely labeled $\mathrm{PO}^{\mathrm{S1}-\text { proj }}$ and $\mathrm{PO}^{\mathrm{M} 1-\text { proj }}$ neurons. As shown in the example traces from a $\mathrm{PO}^{\text {S1-proj }}$ neuron (Fig. $5 B)$, recordings showed large evoked postsynaptic responses with short-term depression as expected for somatosensory and other $\mathrm{PT} \rightarrow \mathrm{TC}$ synapses (Reichova and Sherman, 2004; Groh et al., 2008; Collins et al., 2018). We again sampled and quantified the relative amplitudes of evoked EPSCs for the two cell types (Fig. $5 C)$. In this set of experiments $(n=6$ Rbp4-Cre mice), S1-PT input to $\mathrm{PO}^{\mathrm{S} 1-\text { proj }}$ neurons was substantial (median across 13 cells, $13.2 \pm 13.8 \mathrm{pA}$; median for $n=6$ mice, $39.3 \pm 29.9 \mathrm{pA}$ ), whereas S1-PT to $\mathrm{PO}^{\mathrm{M} 1-\text { proj }}$ neurons was extremely weak (median across 12 cells, $0.5 \pm 0.8 \mathrm{pA}$; median for $n=6$ mice, $1.6 \pm 0.8 \mathrm{pA}$ ) as assessed by both cell-based and animalbased comparisons (unpaired cell-based comparison, $p=0.018$, rank-sum test; pairwise animal-based comparison, $p=$

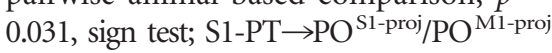
ratio per animal, $25.43 \pm 1.59$, geometric mean \pm geometric standard factor; Fig. 5D-F).

Similar to the CT results described earlier, these results indicate that S1-PT neurons also provide much stronger input to $\mathrm{PO}^{\text {S1-proj }}$ neurons than to $\mathrm{PO}^{\mathrm{M1} \text {-proj }}$ neurons. These electrophysiological findings accord with the anatomical alignment of S1-PT axons with $\mathrm{PO}^{\text {S1-proj }}$ neurons, but not $\mathrm{PO}^{\mathrm{M} 1-p r o j}$ neurons. The findings suggest that the C-T-C circuit formed by $\mathrm{S} 1-\mathrm{PT}$ inputs to $\mathrm{PO}$ is primarily configured as a closed (i.e., $\mathrm{S} 1 \rightarrow \mathrm{PO} \rightarrow \mathrm{S} 1$ ) loop, similar to and in parallel with the CTbased C-T-C circuit in PO (Fig. 5G).

\section{M1-PT input is stronger to M1-projecting than S1-projecting PO neurons}

The finding that S1-PT axons target TC neurons that project back to S1 but not "forward" to M1 raises the question of whether a similar pattern pertains to the thalamic connections of M1-PT axons. Consistent with this possibility, we previously found that $\mathrm{PO}^{\mathrm{M} 1-p r o j}$ neurons are innervated by M1-PT and M1-CT axons (Yamawaki and Shepherd, 2015), but the possibility of connectivity to $\mathrm{PO}^{\mathrm{S1-proj}}$ neurons was not evaluated. We therefore revisited this to better characterize the C-T-C circuits of M1 neurons, focusing on the circuits formed by the PT axons, as these are generally believed to form nonrecurrent, feedforward-type connections in the higher-order thalamus (Sherman and Guillery, 2011). To dissect the cell-type-specific connections in these circuits, we photostimulated ChR2-expressing M1-PT axons in thalamic brain slices while recording from TC neurons identified as projecting to M1 or S1. We injected AAV-DIO-ChR2-YFP in M1 of Rbp4-Cre animals to express ChR2 in M1-PT neurons and 
axons, and injected two different colored tracers into M1 and S1. As described previously, $\mathrm{PO}^{\text {S1-proj }}$ neurons were found somewhat ventral and lateral to $\mathrm{PO}^{\mathrm{M} 1-\text { proj }}$ neurons (Fig. 6A, left). The M1-PT axons overlapped extensively with $\mathrm{PO}^{\mathrm{M} 1-\text { proj }}$ neurons (Fig. 6A, right). With this labeling approach, we targeted whole-cell recordings to $\mathrm{PO}^{\mathrm{M} 1-\text { proj }}$ and $\mathrm{PO}^{\mathrm{S} 1-\text { proj }}$ neurons (Fig. $6 B, C$ ). In this set of experiments ( $n=6 \mathrm{Rbp} 4$-Cre mice), M1-PT input to $\mathrm{PO}^{\mathrm{M} 1-\text { proj }}$ neurons was substantial (median across 10 cells, $50.2 \pm 23.2 \mathrm{pA}$; median for $n=6$ mice, $48.8 \pm 10.4 \mathrm{pA}$ ), whereas M1-PT to $\mathrm{PO}^{\text {S1-proj }}$ neurons was generally very weak (median across 11 cells, $0.9 \pm 0.5 \mathrm{pA}$; median for $n=6$ mice, $0.9 \pm 0.4 \mathrm{pA})$, a statistically significant difference in cell-based and animal-based comparisons (unpaired cell-based comparison, $p=0.0002$, rank-sum test; pairwise animal-based comparison, $p=$ 0.031, sign test; $\mathrm{M} 1-\mathrm{PT} \rightarrow \mathrm{PO}^{\mathrm{M} 1-\text { proj} /}$ $\mathrm{PO}^{\text {S1-proj }}$ ratio per animal, $49.12 \pm 2.44$, geometric mean \pm geometric standard factor; Fig. $6 D-F$ ).

These results indicate that M1-PT neurons provide much stronger input to $\mathrm{PO}^{\mathrm{M} 1-\text { proj }}$ neurons than to $\mathrm{PO}^{\text {S1-proj }}$ neurons, mirroring the findings for S1-PT neurons. These electrophysiological findings again accord with the anatomical alignment of M1-PT axons with $\mathrm{PO}^{\mathrm{M} 1-\text { proj }}$ neurons. Thus, this motor-related C-T-C circuit, similar to its somatosensory counterpart described earlier, is also primarily configured as a recurrent loop (Fig. 6G).

\section{Discussion}

By applying a set of tools combining selective presynaptic and postsynaptic labeling together with optogenetic activation and whole-cell electrophysiological recordings, we dissected the C-T-C circuits of mouse forelimb S1 to determine how CT and PT neurons connect with TC neurons in VPL and PO. Collectively, the results reveal a general theme of multiple recurrent loops, with both CT and PT neurons providing the strongest excitatory input to TC neurons that project back to S1 (Fig. 7).

The CT axonal projections from forelimb S1 to the thalamus overlapped with and formed the strongest excitatory connections to S1-projecting TC neurons in a subregion of VPL. The S1-CT axons also formed moderately strong excitatory connections to recurrently projecting TC neurons in a subregion of PO, forming another loop. Prior studies have shown that some S1-CT neurons, particularly in deeper layer 6, project to PO (Bourassa et al., 1995; Killackey and Sherman, 2003; Chevée et al., 2018); similarly, CT neurons in mouse visual cortex, particularly those in deeper layers, project not only to the lateral geniculate nucleus but also to the matrix-type lateral posterior nucleus (Roth et al., 2016). Our results, delineating recurrent C-T-C loops of S1-CT neurons, are interesting to compare with prior results for $\mathrm{CT}$ neurons in motor areas. Whereas the C-T-C circuits of S1CT neurons in sensory areas generally involve robust CT input to TC neurons in the primary core-type thalamic nucleus (Crandall et al., 2015; Usrey and Sherman, 2019), those of M1-CT neurons involve surprisingly weak connections with M1-projecting TC neurons in VL, which is commonly regarded as the equivalent of a primary core-type nucleus for M1 (Bosch-Bouju et al., 2013; Yamawaki et al., 2014; Yamawaki and Shepherd, 2015). However, similar to S1-CT neurons, M1-CT neurons form robust connections with M1-projecting TC neurons in PO, and those in VM (Yamawaki and Shepherd, 2015). In the premotor-like ALM area, CT projections similarly excite recurrently projecting TC neurons in a subregion of VM (Guo et al., 2018). Thus, for all three areas (S1, M1, ALM), a conserved pattern is that CT neurons excite recurrently projecting TC neurons located in cortical areaspecific subregions of matrix-type thalamic nuclei (PO, VM).

The PT axonal projections from S1 to the thalamus also formed mainly recurrent connections, providing relatively strong excitatory input to $\mathrm{PO}^{\mathrm{S1proj}}$ neurons compared with the S1-PT input to $\mathrm{PO}^{\mathrm{M} 1 \text { proj }}$ neurons, which was largely weak or absent. This latter aspect of our results contrasts with a recent study of sensorimotor pathways in the mouse that described a "transthalamic" circuit from S1-PT neurons to $\mathrm{PO}^{\mathrm{M} 1 \text { proj }}$ neurons (Mo and Sherman, 2019). The apparent discrepancy could reflect differences in methodology and in the anatomical subregions studied, in whisker-related versus forelimb-related cortex and thalamus. Both studies found that S1-PT axons mostly did not overlap with $\mathrm{PO}^{\mathrm{M} 1-\text { proj }}$ neurons. The previous study focused on detecting S1-PT connections to $\mathrm{PO}^{\mathrm{M} 1-\text { proj }}$ neurons, and recordings were accordingly targeted to cells in the narrow overlap zone. In contrast, we focused on measuring the overall relative connec-

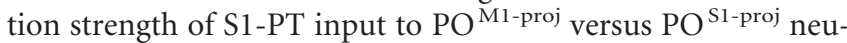
rons and, therefore, recorded from both types of labeled PO neurons, independent of where PT axons were distributed. Our findings are not inconsistent with the main conclusion of Mo and Sherman (2019) that "a circuit exists from layer 5 of S1 through the posterior medial nucleus of the thalamus to M1," insofar as our recordings confirm that such transthalamic connections can be detected, albeit infrequently. In their study, transthalamic connections were not detected in most neurons (70\%), even though recordings were targeted to the overlap zone. Our 
finding of preferential PT innervation of feedback rather than feedforward-projecting neurons in subregions of a matrix-type thalamic nucleus is not without precedent, as we previously observed M1-PT connectivity to $\mathrm{PO}^{\mathrm{Mlproj}}$ neurons (albeit without comparison with other types of thalamic projection neurons, as was done here; Yamawaki and Shepherd, 2015) and recently found the same organization for PT inputs to VM from two motor areas, M1 and ALM, which form separate loops through subregions of VM (Guo et al., 2018); PFC-PT projections also innervate VM, in addition to MD (Collins et al., 2018). We emphasize that our approach, though well suited for our main goal of testing connectivity to recurrently projecting TC neurons and certainly capable of detecting transthalamic connections (Yamawaki et al., 2016a), is not designed to fully characterize input to the type of dual-projecting TC neurons that would mediate transthalamic connectivity. This is because the approach relies on targeting two specific cortical areas for retrograde labeling of TC neurons, which is reliable for the recurrent projections but could miss the nonrecurrent branches. For the forelimb S1-projecting PO neurons studied here, we targeted adjacent forelimb M1 as a functionally relevant candidate site of dual branches from their axonal arbors, but such branches could have been located elsewhere in a highly focal manner. Consistent with this possibility, anatomical evidence supports the idea that at least some TC axons branch in two or more cortical areas in a dual or multifocal pattern, rather than a broadly diffuse manner (Kuramoto et al., 2009, 2015; Clascá et al., 2012; Ohno et al., 2012; RodriguezMoreno et al., 2020; Winnubst et al., 2019).

Our findings support and extend patterns observed in prior anatomical studies of cortical projections to the thalamus from various cortical locations. In particular, anatomical characterization of CT and PT projections from vibrissal M1 and M2 (Jeong et al., 2016) showed that thalamic branching patterns from the two areas tend to be adjacent and segregated, but those from CT and PT neurons in the same area tend to be overlapping and similar. Results from a recent large-scale analysis of cortical projections to the thalamus suggest that these patterns, with different areas having distinct patterns of thalamic projections but, in most cases, with substantial overlap of the CT and PT components, may be widespread (Harris et al., 2019). Our findings, suggesting largely parallel segregated projections from forelimb S1 and M1 to different subregions of $\mathrm{PO}$, with mainly convergent rather than divergent CT and PT components in both cases, appear to fit this general pattern.

Do the C-T-C circuits of the mouse somatosensory and motor pathways share common organizing principles, or are they specialized according to the particular behavioral functions mediated by S1, M1, and ALM? Although our results do not resolve this issue, a number of similarities across circuits suggest the outlines of a general circuit-level model. In particular, for the matrix-type thalamic nuclei (PO, VM), the available evidence indicates that both the CT and PT neurons in a given cortical area (S1, M1, ALM) project in a similar manner to specific nuclear subregions, each of which contains TC neurons that project back to the same cortical area it receives axons from. Both CT and PT axons form relatively strong excitatory connections to the recurrently projecting TC neurons in those nuclear subregions. This model also accords with evidence that nuclei such as PO are not homogeneous nuclei but rather complexes composed of multiple subdivisions (Jones, 2007). For the primary core-type nuclei, a lack of PT innervation appears to be the rule for the sensory cortex (Sherman and Guillery, 2011) and M1 (Yamawaki and Shepherd, 2015). Furthermore, the extent of CT innervation to form feed- back connections in core-type nuclei appears to be systemspecific, with recurrent circuits being relatively strong in somatosensory pathways $\left(\mathrm{S} 1-\mathrm{CT} \rightarrow \mathrm{VPL}^{\mathrm{S} 1-\text { proj }}\right.$ neurons; this study) but weak in motor pathways (M1-CT $\rightarrow \mathrm{VL}^{\mathrm{M} 1-\text { proj }}$ neurons; Yamawaki and Shepherd, 2015). This may reflect a fundamental difference in the underlying organization of sensory pathways, where cortical feedback is thought to play a major role in attentional and other perception-related processing, versus motor pathways, where cerebellar output appears to be conveyed in a largely unidirectional manner to M1. Regarding the recurrent C-T-C loops of PT neurons, another speculative possibility is that, whereas the synaptic connectivity appears similarly configured, the circuits nevertheless mediate distinct specialized functions in the different areas and systems. Whereas the PTmediated C-T-C loops of ALM $\leftrightarrow \mathrm{VM}$ are implicated in supporting persistent activity associated with action planning (Guo et al., 2017, 2018), those of $\mathrm{S} 1 \leftrightarrow \mathrm{PO}$ and $\mathrm{M} 1 \leftrightarrow \mathrm{PO}$ may be involved in sensory perception and motor execution, respectively. Our results, by delineating the cellular connectivity in S1associated C-T-C circuits, provide a framework for targeted investigation of how C-T-C circuit activity relates to the neural computations underlying sensorimotor behavioral functions.

\section{References}

Auffret M, Ravano VL, Rossi GMC, Hankov N, Petersen MFA, Petersen CCH (2018) Optogenetic stimulation of cortex to map evoked whisker movements in awake head-restrained mice. Neuroscience 368:199-213.

Bortone DS, Olsen SR, Scanziani M (2014) Translaminar inhibitory cells recruited by layer 6 corticothalamic neurons suppress visual cortex. Neuron 82:474-485.

Bosch-Bouju C, Hyland BI, Parr-Brownlie LC (2013) Motor thalamus integration of cortical, cerebellar and basal ganglia information: implications for normal and parkinsonian conditions. Frontiers in computational neuroscience 7:163.

Bourassa J, Pinault D, Deschênes M (1995) Corticothalamic projections from the cortical barrel field to the somatosensory thalamus in rats: a single-fibre study using biocytin as an anterograde tracer. Eur J Neurosci 7:19-30.

Chevée M, Robertson JJ, Cannon GH, Brown SP, Goff LA (2018) Variation in activity state, axonal projection, and position define the transcriptional identity of individual neocortical projection neurons. Cell Rep 22: 441-455.

Clascá F, Rubio-Garrido P, Jabaudon D (2012) Unveiling the diversity of thalamocortical neuron subtypes. Eur J Neurosci 35:1524-1532.

Collins DP, Anastasiades PG (2019) Cellular specificity of cortico-thalamic loops for motor planning. J Neurosci 39:2577-2580.

Collins DP, Anastasiades PG, Marlin JJ, Carter AG (2018) Reciprocal circuits linking the prefrontal cortex with dorsal and ventral thalamic nuclei. Neuron 98:366-379.e4

Crandall SR, Cruikshank SJ, Connors BW (2015) A corticothalamic switch: controlling the thalamus with dynamic synapses. Neuron 86:768-782.

Deschênes M, Bourassa J, Pinault D (1994) Corticothalamic projections from layer $\mathrm{V}$ cells in rat are collaterals of long-range corticofugal axons. Brain Res 664:215-219.

Deschênes M, Veinante P, Zhang ZW (1998) The organization of corticothalamic projections: reciprocity versus parity. Brain Res Brain Res Rev 28:286-308.

Ferezou I, Haiss F, Gentet LJ, Aronoff R, Weber B, Petersen CC (2007) Spatiotemporal dynamics of cortical sensorimotor integration in behaving mice. Neuron 56:907-923.

Gerfen CR, Paletzki R, Heintz N (2013) GENSAT BAC cre-recombinase driver lines to study the functional organization of cerebral cortical and basal ganglia circuits. Neuron 80:1368-1383.

Gong S, Doughty M, Harbaugh CR, Cummins A, Hatten ME, Heintz N, Gerfen CR (2007) Targeting cre recombinase to specific neuron populations with bacterial artificial chromosome constructs. J Neurosci 27: 9817-9823.

Grant E, Hoerder-Suabedissen A, Molnár Z (2016) The regulation of corticofugal fiber targeting by retinal inputs. Cereb Cortex 26:1336-1348. 
Groh A, de Kock CP, Wimmer VC, Sakmann B, Kuner T (2008) Driver or coincidence detector: modal switch of a corticothalamic giant synapse controlled by spontaneous activity and short-term depression. J Neurosci 28:9652-9663.

Guo ZV, Inagaki HK, Daie K, Druckmann S, Gerfen CR, Svoboda K (2017) Maintenance of persistent activity in a frontal thalamocortical loop. Nature 545:181-186.

Guo K, Yamawaki N, Svoboda K, Shepherd GMG (2018) Anterolateral motor cortex connects with a medial subdivision of ventromedial thalamus through cell type-specific circuits, forming an excitatory thalamocortico-thalamic loop via layer 1 apical tuft dendrites of layer 5B pyramidal tract type neurons. J Neurosci 38:8787-8797.

Harris JA, Mihalas S, Hirokawa KE, Whitesell JD, Choi H, Bernard A, Bohn P, Caldejon S, Casal L, Cho A, Feiner A, Feng D, Gaudreault N, Gerfen CR, Graddis N, Groblewski PA, Henry AM, Ho A, Howard R, Knox JE, et al. (2019) Hierarchical organization of cortical and thalamic connectivity. Nature 575:195-202.

Hoerder-Suabedissen A, Hayashi S, Upton L, Nolan Z, Casas-Torremocha D, Grant E, Viswanathan S, Kanold PO, Clasca F, Kim Y, Molnár Z (2018) Subset of cortical layer $6 \mathrm{~b}$ neurons selectively innervates higher order thalamic nuclei in mice. Cereb Cortex 28:1882-1897.

Hooks BM, Mao T, Gutnisky DA, Yamawaki N, Svoboda K, Shepherd GMG (2013) Organization of cortical and thalamic input to pyramidal neurons in mouse motor cortex. J Neurosci 33:748-760.

Jeong M, Kim Y, Kim J, Ferrante DD, Mitra PP, Osten P, Kim D (2016) Comparative three-dimensional connectome map of motor cortical projections in the mouse brain. Sci Rep 6:20072.

Jones EG (2007) The thalamus, Ed 2. Cambridge, UK: Cambridge UP.

Joshi A, Middleton JW, Anderson CT, Borges K, Suter BA, Shepherd GMG, Tzounopoulos T (2015) Cell-specific activity-dependent fractionation of layer $2 / 3->5 B$ excitatory signaling in mouse auditory cortex. J Neurosci 35:3112-3123.

Killackey HP, Sherman SM (2003) Corticothalamic projections from the rat primary somatosensory cortex. J Neurosci 23:7381-7384.

Kim J, Matney CJ, Blankenship A, Hestrin S, Brown SP (2014) Layer 6 corticothalamic neurons activate a cortical output layer, layer $5 \mathrm{a}$. J Neurosci 34:9656-9664.

Kuramoto E, Furuta T, Nakamura KC, Unzai T, Hioki H, Kaneko T (2009) Two types of thalamocortical projections from the motor thalamic nuclei of the rat: a single neuron-tracing study using viral vectors. Cereb Cortex 19:2065-2077.

Kuramoto E, Ohno S, Furuta T, Unzai T, Tanaka YR, Hioki H, Kaneko T (2015) Ventral medial nucleus neurons send thalamocortical afferents more widely and more preferentially to layer 1 than neurons of the ventral anterior-ventral lateral nuclear complex in the rat. Cereb Cortex 25: 221-235.

Lein ES, Hawrylycz MJ, Ao N, Ayres M, Bensinger A, Bernard A, Boe AF, Boguski MS, Brockway KS, Byrnes EJ, Chen L, Chen L, Chen TM, Chin MC, Chong J, Crook BE, Czaplinska A, Dang CN, Datta S, Dee NR, et al. (2007) Genome-wide atlas of gene expression in the adult mouse brain. Nature 445:168-176.

Li X, Yamawaki N, Barrett JM, Körding KP, Shepherd GMG (2018) Scaling of optogenetically evoked signaling in a higher-order corticocortical pathway in the anesthetized mouse. Front Syst Neurosci 12:16.
Madisen L, Zwingman TA, Sunkin SM, Oh SW, Zariwala HA, Gu H, Ng LL, Palmiter RD, Hawrylycz MJ, Jones AR, Lein ES, Zeng H (2010) A robust and high-throughput cre reporting and characterization system for the whole mouse brain. Nat Neurosci 13:133-140.

Mo C, Sherman SM (2019) A sensorimotor pathway via higher-order thalamus. J Neurosci 39:692-704.

Ohno S, Kuramoto E, Furuta T, Hioki H, Tanaka YR, Fujiyama F, Sonomura T, Uemura M, Sugiyama K, Kaneko T (2012) A morphological analysis of thalamocortical axon fibers of rat posterior thalamic nuclei: a single neuron tracing study with viral vectors. Cereb Cortex 22:2840-2857.

Peron SP, Freeman J, Iyer V, Guo C, Svoboda K (2015) A cellular resolution map of barrel cortex activity during tactile behavior. Neuron 86:783-799.

Reichova I, Sherman SM (2004) Somatosensory corticothalamic projections: distinguishing drivers from modulators. J Neurophysiol 92: 2185-2197.

Rodriguez-Moreno J, Porrero C, Rollenhagen A, Rubio-Teves M, CasasTorremocha D, Alonso-Nanclares L, Yakoubi R, Santuy A, MerchanPérez A, DeFelipe J, Lübke JHR, Clasca F (2020) Area-specific synapse structure in branched posterior nucleus axons reveals a new level of complexity in thalamocortical networks. J Neuroscience. Advance online publication. Retrieved February 13, 2020. doi: 10.1523/JNEUROSCI. 2886-19.2020

Roth MM, Dahmen JC, Muir DR, Imhof F, Martini FJ, Hofer SB (2016) Thalamic nuclei convey diverse contextual information to layer 1 of visual cortex. Nat Neurosci 19:299-307.

Sherman SM, Guillery RW (2011) Distinct functions for direct and transthalamic corticocortical connections. J Neurophysiol 106:1068-1077.

Shibuki K, Hishida R, Murakami H, Kudoh M, Kawaguchi T, Watanabe M, Watanabe S, Kouuchi T, Tanaka R (2003) Dynamic imaging of somatosensory cortical activity in the rat visualized by flavoprotein autofluorescence. J Physiol 549:919-927.

Suter BA, O’Connor T, Iyer V, Petreanu LT, Hooks BM, Kiritani T, Svoboda K, Shepherd GMG (2010) Ephus: multipurpose data acquisition software for neuroscience experiments. Front Neural Circuits 4:100.

Usrey WM, Sherman SM (2019) Corticofugal circuits: communication lines from the cortex to the rest of the brain. J Comp Neurol 527:640-650.

Winnubst J, Bas E, Ferreira TA, Wu Z, Economo MN, Edson P, Arthur BJ, Bruns C, Rokicki K, Schauder D, Olbris DJ, Murphy SD, Ackerman DG, Arshadi C, Baldwin P, Blake R, Elsayed A, Hasan M, Ramirez D, Dos Santos B, et al. (2019) Reconstruction of 1,000 projection neurons reveals new cell types and organization of long-range connectivity in the mouse brain. Cell 179:268-281.e213.

Yamawaki N, Shepherd GMG (2015) Synaptic circuit organization of motor corticothalamic neurons. J Neurosci 35:2293-2307.

Yamawaki N, Borges K, Suter BA, Harris KD, Shepherd GMG (2014) A genuine layer 4 in motor cortex with prototypical synaptic circuit connectivity. eLife 3:e05422.

Yamawaki N, Radulovic J, Shepherd GMG (2016a) A corticocortical circuit directly links retrosplenial cortex to M2 in the mouse. J Neurosci 36:9365-9374.

Yamawaki N, Suter BA, Wickersham IR, Shepherd GMG (2016b) Combining optogenetics and electrophysiology to analyze projection neuron circuits. Cold Spring Harb Protoc 2016:pdb prot090084. 